\title{
Interactive comment on "The Role of Sediment-induced Light Attenuation on Primary Production during Hurricane Gustav (2008)" by Zhengchen Zang et al.
}

\section{Zhengchen Zang et al.}

zxue@lsu.edu

Received and published: 12 May 2020

We would like to thank you for your constructive comments, which are very helpful and are/will be addressed in the revision.

RC1 point 1: The experiment design is not flawless. It is not explained why a 20year run was necessary to achieve a stable initial condition, which itself is not shown or validated against data at all. Then, the model starts a few days before the hurricane makes landfall, but it would have made more sense to start a week earlier to show what a "normal" state of the ecosystem in that time period would have looked like. Also, to show whether the model actually recovers to said "normal" state, the model should 
have been run a week longer after it actually ends. While this would have taken more time, a 20y run to spin up the ecosystem was strictly speaking not necessary. 2-5 years are common in literature, and setting this up is literally an issue of an hour, if the forcing is already available.

Authors: Our experience in coupled biophysical modeling is that the biological model need several years to achieve a steady state. As our model is forced by global HyCOM model for physics boundary condition, we initiated our model from 1993 when the earliest HyCOM results is available. After 16 years of spin up (1993-2008), we believe that it is reasonable to extract its result as the initial condition for our hurricane simulation. To validate the initial condition, we will add one more panel in Fig. 3 and compare with satellite result.

Starting the model one week earlier is very challenging in hurricane simulation because the low-pressure vortex of hurricane is not formed yet. We tried to extend our simulation one week longer, but the water did not recover to normal condition because another hurricane, Ike, approached the study region, and vertical mixing turned to be strong again before stratification fully recovered to normal condition.

RC1 point 2: In line 72, parameterisation approaches to sediment specific attenuation are discussed. One might mention the strong correlation between sediment content and bathymetry, as employed or discussed e.g. in Zhou et al. 2017 (https://doi.org/10.1016/j.pocean.2017.10.008) and Thewes et al. 2020 (https://doi.org/10.3389/fmars.2019.00816). Their approach has the disadvantage of not being time variant, making a stronger case for online modelling of sediment.

Authors: The two studies use a simplified scheme i/N (i represents the vertical level and $\mathrm{N}$ is the total number of vertical layer) to parametrize sediment-induced light attenuation. We will cite the two new papers in our introduction.

RC1 point 3: Section 2.1 This section heavily relies on previous publications, which is fine. However, this way, a few bits of information fall under the radar, like how many

Printer-friendly version

Discussion paper 
different sediment types there are. Also, it is possible and not too difficult to program a specific coefficient for each sediment type. An explanation as to why this was not done is needed.

Authors: We will add more information about sediment setup in section 2.1.

Applying a specific coefficient for each sediment type is not challenging. However, that cannot help us better understand the role of sediment in the underwater optic environment because: 1 ) sediment light attenuation coefficient is not only determined by size, and we don't have any measurements about light attenuation coefficients for different types of sediment, so the selection of attenuation coefficients might be arbitrary; 2) resolving the fraction of different types of sediment in the water column during the hurricane is still a challenge due to the complexity of sediment dynamics (flocculation and deflocculating; hindered settling effect, sea floor consolidation and liquefaction, etc.). In addition, sediment fraction is not calibrated in our sediment model paper (Zang et al., 2018) because no observation is available. Thus, using different coefficient for each type of sediment might potentially introduce more uncertainties but little useful information. We plan to stick to our original scheme, which uses one constant coefficient for all sediments. Of course, this coefficient only represents the "mean" value of attenuation coefficient for all types of sediment.

RC1 point 4: In line 110, it says the model was "largely built on" NEMURO. Other than the addition of a sediment specific attenuation term, has anything else been changed?

Authors: The original NEMURO model does not have chlorophyll, which is not in favor of model validation. We include chlorophyll in our model following Fennel et al. (2006).

$\mathrm{RC} 1$ point 5: Because passages in later sections are on the subject of hypoxia, NEMURO might not have been an ideal choice. Perhaps it should be explained why NEMURO was chosen over models that have been coupled to ROMS and published before, which calculate oxygen as a state variable.

Printer-friendly version

Discussion paper 
Authors: We use NEMURO because it includes diatom, which accounts for $20-60 \%$ of total phytoplankton biomass (Zhao and Quigg, 2014) in the northern Gulf of Mexico. Since simulating hypoxia is not the objective of this study, not including oxygen dynamics in NEMURO should not harm our conclusion.

RC1 point 6: There is a very important error in the equation in line 132. Because plankton biomass and sediment content are functions of depth, the equation must include integrals of PSn + PLn and SSC over depth. Section 2.2 The authors should explain why they selected the specific values of a_sed that they chose. Are the studies to which they refer conducted in the same region? Are they at all comparable to the model situation?

Authors: We will update the equation in section 2.1. In the benchmark run, $\alpha \_$sed=0.059 is based on the study in the Delaware Bay (McSweeney et al., 2017) because no studies provide a specific light attenuation coefficient of sediment in the nGoM. Several studies estimated $\alpha \_$sed in the nGoM but only gave a wide range of $\alpha \_$sed from 10-3 to 100, which cannot be applied to our numerical study because we need a specific number rather than a range. We will re-design the sensitivity tests following reviewer's suggestion in Question 17.

RC1 point 7: Section 3 The authors have conducted a 20y run to obtain an initial condition, yet the initial condition is never shown or validated against data. Because the $20 \mathrm{y}$ run is not the object of the study, it needs no validation, but the initial condition certainly does. This could be an extra panel in figure 3.

Authors: We plan to add an extra panel in Fig. 3 to show the initial condition (chlorophyll) of our model. RC1 point 8: RMSE and R were computed. For this to be done, one of the data sources would have to be regridded to match the other. Was satellite data or model data regridded?

Authors: We interpolate satellite data to model grid to make the comparison.

Printer-friendly version

Discussion paper 
RC1 point 9: Figure 4 shows logarithmic values for SeaWiFS and model data. Were RMSE and $R$ calculated using the actual data or the logarithmic data?

Authors: Both RMSE and R are calculated using the logarithmic data.

RC1 point 10: In line 174 it says that the "model's performance was significantly improved in high productivity waters where chlorophyll concentration is $>1 \mathrm{mg} / \mathrm{m3}$ ". Does this refer to satellite or model data?

Authors: This refers to both model and observed data (see line 203).

RC1 point 11: Section 4.1 NPP is not defined in the text. Although there are literature definitions, this should be explained.

Authors: We will define net primary production (NPP) at the very beginning of section 4.1 .

RC1 point 12: Why is there a 3-4hour delay in chlorophyll with respect to NPP? Are biomass and chlorophyll uncoupled? Do different species have different $\mathrm{C}: \mathrm{CHL}$ ratios?

Authors: The peak of NPP suggests the maximum growth rate of chlorophyll, not the peak of chlorophyll. Chlorophyll concentration reached its maximum when NPP went down and was compensated by other loss terms (e.g., grazing and mortality). In this study, we estimate chlorophyll concentration following Fennel et al. (2006), and the variation of chlorophyll due to the growth of small and large phytoplankton are simulated individually, so the $\mathrm{C}: \mathrm{Chl}$ ratio of different types of phytoplankton are not the same in the model.

RC1 point 13: In line 197, it says that surface cooling and decreased light contributed to reductions of chlorophyll and NPP. Can you identify the individual contributions? From fig. $5 b$, it seems that surface cooling limits by about a factor of 0.5 and light limits by up to 0.3 at the maximum. This should be calculable just by putting in representative values in the respective equations for light and temperature limitation. Also, it says reduced temperature and light availability "contributed" to the reductions. What else

Printer-friendly version

Discussion paper




might have contributed? The first peak in figure $5 \mathrm{a}$ is significantly broader than most of the following peaks. After that, they seem to have an almost bimodal quality. This is not addressed in the text. Is this due to the different species? It is true for both runs. The NPP in test 1 recovers almost immediately to the same peak value, albeit narrower. Chlorophyll does not really recover to pre-storm values at all, but the benchmark run does. Again, is this due to speciation?

Authors: In the model, the growth of phytoplankton (Gpp) is determined by temper-

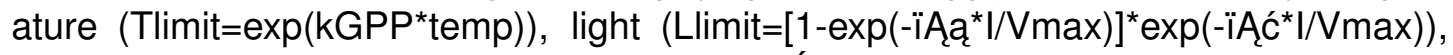
and nutrient (Nlimit=NO3/(NO3+KNO3)exp(-ïAZŹNH4)+NH4/(NH4+KNH4)):

\section{Gpp=Gppmax* Tlimit* Llimit* Nlimit}

It is noteworthy that temperature-limit factor (Tlimit) is different from light- and nutrientlimit factors (Llimit and Nlimit): Tlimit is much larger than 1, while Llimit and Nlimit are between 0 and 1 . Thus, Tlimit, Llimit and Nlimit are not comparable quantitatively. However, Tlimit declines with lower temperature, and Llimit decreases with lower light availability (I), so it is safe to conclude that reduced temperature and light contribute to the lower primary production.

Although NEMURO model is able to resolve phytoplankton size structure, we have no field data to convince the readers that our model is able to well reproduce the fraction of different types of phytoplankton. Therefore, we decide not to analyze small or large phytoplankton separately in this study.

RC1 point 14: I suggest to separate contributions of the individual plankton species to both NPP and CHL. It looks to me as though one of the two phytoplankton species is more susceptible to light or temperature limitation than the other. If the run had been longer by a week in both directions, one might have seen a full recovery to a "usual" state after the hurricane (i.e. the broad peak in NPP on the 30th of August). It looks like the benchmark run recovers faster to that broad, supposedly "normal" peak, while test 1 shows a quicker recovery in the leading peak. It may, as stated in the manuscript, be

Printer-friendly version

Discussion paper 
largely due to the boost in NO3. However, temperature and light sensitivity might be different for the two phytoplankton species. This should be disclosed.

Authors: As mentioned above (Question 13), we are very prudent about simulated phytoplankton size structure due to the lack of observation, so we do not have plan to provide detailed analysis regarding different types of phytoplankton.

RC1 point 15: NPP is a depth integrated quantity, but only surface chlorophyll is shown. There is no info on lower layer productivity. What is the vertical structure of phytoplankton, or rather, does it change when switching on sediment specific attenuation? Section 4.2 Figure 6 is very illustrative. It might be helpful to have two more panels showing NO3. In that case, perhaps rotate orientation to columns <-> time and rows <-> parameter.

Authors: We will include NO3 profiles of the benchmark run and test 1 in Fig. 6 and adjust the orientation of this figure.

RC1 point 16: In the second paragraph of this section, the authors discuss hypoxia. However, NEMURO does not provide oxygen output. Then, the situation in the nGoM is compared to the Delaware estuary. It would be prudent to show that these areas are at all comparable, which they are likely not, because an estuary is usually bounded horizontally and is characterised by strong lateral salinity gradients. The Delaware estuary experiences hypoxia due to density stratification. There is no figure representing temperature or salinity stratification in the nGoM model, but only chlorophyll and SSC. The latter strongly influences the former and SSC stratification is perhaps purely due to sediment settling. What is implied in the text is that the chlorophyll stratification is due to a density stratification, and that phytoplankton does not reach the lower layers, because it is physically bound. There needs to be a figure showing temperature and salinity, or density and stability frequency, to be able to imply a similar situation as in the Delaware estuary. In line 237 of the text it says that "post-hurricane stratification recovery" prevented oxygen ventilation to the bottom. Is this with reference to the model

Printer-friendly version

Discussion paper 
in the study? Please show stratification along the transect. Perhaps show some in situ data of oxygen from that time period, to show that there actually was a hypoxia event after Gustav. Otherwise, consider removing the paragraph.

Authors: We will add water density anomaly profile in Fig. 7 to illustrate the variation of vertical structure. In our sediment paper (Zang et al. 2018), the analysis of Brunt Väisälä frequency (BVF) also demonstrate the recovery of stratification after hurricane. In addition, both previous field measurements (McCarthy et al., 2013) and model results (Moriarty et al., 2018) suggest that water stratification after strong resuspension event is favorable to the formation of hypoxia, so we prefer to keep this paragraph in the updated manuscript.

RC1 point 17: Section 4.4 Although it is clear from a modeler's perspective and from a methodical point of view why the authors decide to do a sensitivity study with regards to sediment specific attenuation, the explanation that this is to accommodate for the way different types of sediment attenuate light is vague. It is true that different types attenuate differently, but then it appears more reasonable to simply compute the model with one coefficient for each sediment type. The programming effort is a day's work at best. The researching effort to get plausible values might be a little more work, but all in all it is not clear why this has not been done. It might be worth an explanation or at least an elaboration as to what prevented the authors from doing that. Again, it is a perfectly reasonable approach to perform a sensitivity study over a varying singular attenuation coefficient, yet the explanation lacks context. Why were the specific values chosen? They were taken from referenced studies, but are the sediments in these studies comparable in their make up (see comments to section 2)? Why not linearly vary around a reasonable value by $20 \%$ (this is almost the case anyway)? Why not do 5 tests instead of 3 ?

Authors: We will re-design four sensitivity tests (tests 2-5) by increasing (decreasing) attenuation coefficient by 20 and $40 \%$, respectively. 
$\mathrm{RC} 1$ point 18: Figure 7 hints at the chaotic nature of the ecosystem by showing how a small change in an initial state can alter the following development. Even though after the 6th of September, SSC at the surface was almost zero, the benchmark and test 2 deviate more from each other after that day than they do before. This is perhaps an interesting point to make.

Authors: Our discussion in section 4.4 explains why our model is more sensitive to $\alpha \_$sed when SSC is in a certain range. The contribution of high SSC overwhelms that of $\alpha \_$sed to the variation of sediment-induced light attenuation term ( $\alpha \_$sed SSC), while light attenuation term is very small when SSC is almost zero. Therefore, the primary production and nutrient dynamics are only sensitive to $\alpha$ sed when SSC is moderately high, and that explains why we conclude there is a range for SSC in which $\alpha$ sed plays a vital role in photosynthesis (see our response to Question 20).

RC1 point 19: Ideally, for all runs with sediment attenuation, the light limitation should be identical over time. However, given the chaotic nature of turbulence, an elaboration as to whether or not the individual run's SSC deviate from each other in any way is missing. It is not expected to deviate heavily, but a note on whether or not there is deviation is appropriate. Authors: We will elaborate that the derivation due to turbulence was not considered in this study.

RC1 point 20: In line 290 it says that the study does not provide a widely accepted SSC range to determine whether a_sed plays a vital role in photosynthesis and primary production [...]. This is somewhat confusing, as a_sed clearly plays a vital role. If it is 0 , the model output looks drastically different in some regards. I am not sure what is meant by a "widely accepted SSC range". Does that mean that a certain value of a_sed may be valid for a certain range of SSC? If so, please rephrase the sentence to make this clearer.

Authors: As shown in the sensitivity tests, primary production was not sensitive to $\alpha \_$sed when SSC is too low (pre-hurricane) or too high (hurricane landing), which

Printer-friendly version

Discussion paper 
means there is a range of SSC in which the ecosystem is sensitive to $\alpha$ sed. Due to the differences (hydrodynamics, phytoplankton species, nutrient sources, optic feature of sediment particles, etc.) between coastal ecosystems, this range might vary greatly in different systems. Therefore, we state that "this study does not provide a widely accepted SSC range in which $\alpha$ sed plays a vital role in photosynthesis and primary production".

Interactive

RC1 point 21: The authors further speculate in line $296 \mathrm{ff}$ that there might be variation on possibly annual to decadal scales. To emphasise the validity of the claim, as well as the importance of the subject, the following studies on the North Sea might be of interest and inspiration: Dupont \& Aksnes 2013 (https://doi.org/10.1016/j.ecss.2013.08.010), Capuzzo et al. 2015 (https://doi.org/10.1111/gcb.12854) and Wilson \& Heath 2019 (https://doi.org/10.5194/os-15-1615-2019). It might also be noteworthy that hurricane events are predicted to occur more frequently under the changing climate. Implications of this could be lowered primary production.

Authors: These studies confirmed the importance of long-term sediment dynamics in marine ecosystem and biogeochemical cycling, so we included these papers in our manuscript to support our speculation. The frequency of hurricane events under the changing climate in the future is still controversial because different prediction models show opposite results: the numerical study of Bender et al. (2010) found the overall frequency of Atlantic hurricanes decrease due to global warming, while Knutson and Tuleya (2004) suggested the increase frequency of hurricane due to CO2-induced warming. Thus, we decide not to discuss the influence of hurricane frequency on primary production.

$\mathrm{RC} 1$ point 22: Section 4.5 The authors are right in addressing the lack of CDOM representation, and they correctly point out that many models share the same weakness. However, as they also point out, salinity is often used as a proxy for CDOM via an inverse relationship, which is well documented and easy to implement. It does make the

Printer-friendly version

Discussion paper 
paper more complex, but it might have been worth the effort, specifically when doing a sensitivity analysis with respect to when an ecosystem shifts from a nutrient limited to a light limited regime. The CDOM contribution would undoubtedly have biased the results of the sensitivity test. It is therefore appropriate to include a sentence or two in the discussion, explaining that CDOM specific attenuation would likely shift a hypothetical threshold a_sed downwards, below which the regime may be considered nutrient limited.

Authors:Although the Mississippi-Atchafalaya River is one of the major sources of CDOM to the nGoM due to its high discharge, previous field measurements found that coastal wetlands provide important CDOM contributions to nearshore shelf waters, and the relationship between CDOM concentration and salinity off the wetlands was totally different from that in the river plume (Chen and Gardner, 2004; Shank and Evans, 2011). Moreover, our model does not include freshwater discharge from wetlands. Therefore, using an inverse relationship between salinity and CDOM to estimate its light attenuation is not applicable in our study region. In our model uncertainty discussion (section 4.5 lines 345-348), we will add the potential impacts of neglecting CDOM light attenuation on our model results following reviewer's suggestion.

$\mathrm{RC} 1$ point 23: Section 5 The authors say in the first sentence of this section that they "introduced a sediment-induced light attenuation algorithm to the coupled physicalbiogeochemical model on the platform of ROMS", which is, strictly speaking, not true. They did introduce it to NEMURO, however, ROMS still uses a different light attenuation scheme. See e.g. Mobley et al. 2015 (https://doi.org/10.1002/2014JC010588) or Cahill et al. 2008 (https://doi.org/10.1029/2008GL033595) to find what the not insignificant difference is. There is no coupling between sediments and light absorption in the physical model.

Printer-friendly version

Authors:We will change the sentence to "We introduced a sediment-induced light attenuation algorithm to the biogeochemical model on the platform of ROMS". In addition, we will cite these two papers in the first section (Introduction) and highlight that

Discussion paper 
our model does not modify water heating due to light absorption in the hydrodynamic model.

RC1 point 24: In line 367 , the authors again claim that the post hurricane situation might have caused a hypoxia. Without any data, this is purely speculative and does not belong in the conclusions chapter.

Authors: We will remove the statement about hypoxia in the conclusion chapter.

RC1 point 25: Minor comments see attached documents for grammar errors and other minor comments.

Authors: We will correct the grammar errors and update our manuscript following the reviewer's comments.

Interactive comment on Biogeosciences Discuss., https://doi.org/10.5194/bg-2020-58, 2020. 